\title{
Three-dimensional extremely short optical pulses in graphene with coulomb impurities with taking into account inhomogeneity
}

\author{
N. N. Konobeeva ${ }^{1}$, A. A. Polunina ${ }^{1}$, M. B. Belonenko ${ }^{1,2}$ \\ ${ }^{1}$ Volgograd State University, 400062, Volgograd, Russia \\ ${ }^{2}$ Volgograd Institute of Business, 400048, Volgograd, Russia \\ yana_nn@inbox.ru
}

DOI 10.17586/2220-8054-2016-7-6-936-940

\begin{abstract}
Maxwell's equations were considered for the electromagnetic field propagating in doped graphene taking spatial inhomogeneity in the threedimensional case. The electronic spectrum for the graphene subsystem was obtained from the model taking into account the Coulomb impurity. The effective equation for the vector potential of the electromagnetic field was solved numerically. A comparison of the forms of extremely short optical pulses is done for the case with recording inhomogeneities and one without.
\end{abstract}

Keywords: optical pulses, Coulomb impurities, inhomogeneity.

Received: 10 April 2016

Revised: 16 May 2016; 7 June 2016

\section{Introduction}

Recently interest in the study of extremely short optical pulses in different nanoobjects has increased, from both theoretical and practical viewpoints [1-7]. This is primarily due to the prospects of using such pulses to solve different kinds of problems which occur in modern nanoelectronics. In this paper, we will study features of the three-dimensional propagation of extremely short pulses (ESP) in doped graphene with consideration of its inhomogeneity. We mean that inhomogeneity is the region of high electron density. Random inhomogeneity of this field (due to the random arrangements of impurities) can result in interesting and unexpe [8] is related to the two-dimensional simulation of the propagation of ultrashort electromagnetic pulses in an array of CNTs (carbon nanotubes) with the inhomogeneity of the field along the axis (in this case, the field is not randomly inhomogenous, we assume that the inhomogeneity is given experimentally). In addition, in recent years authors conducted a comprehensive study of the last task in the 3D case, as a result of which, have demonstrated the possibility of bipolar propagation of electromagnetic breathers through an array of CNT with consideration of field inhomogeneities [9]. In this case, the electromagnetic pulse causes a significant redistribution of electron density in the sample for both 2D, and 3D systems. Besides field inhomogeneities that lead to a redistribution of the electrons, there are other natural inhomogeneities observed in experimental samples. This is especially important in the case where the inhomogeneities are caused by increased concentration regions of conduction electrons due to the presence of impurities. It is worth noting that the existence of different kinds of impurities and the nature of their interactions with graphene also requires the use of a variety of approaches. For example, in [10], the investigators considered the case of impurities in graphene with the disorder, where the spectrum of electrons was determined from the renormalization group approach.

In this paper, we will rely on a different approach [11], which is to consider the Coulomb charged impurities in graphene. In the cited paper, the effect of the gap, which is induced with impurities, was studied by using the Hartree-Fock method for the calculation of the Fermi velocity. This led to their own energy corrections to the polarization. The result is a renormalized Fermi velocity, which has a logarithmic dependency on the electron's energy.

\section{The statement of the problem and basic equations}

Consider the propagation of extremely short electromagnetic pulses in doped graphene with impurities, with consideration of the ultimate quasiparticle mass (i.e. with the gap in the spectrum). The vector-potential $A$ is directed along the graphene sheet, the direction of the electric field propagation is perpendicular to the graphene sheet. Graphene is oriented in the plane XOY.

The dispersion relation for graphene can be found according to well-known relationship of charges speed and energy of electrons: $\nu_{y}=\frac{\partial \varepsilon\left(q_{x}, q_{y}\right)}{\partial q_{y}}$. 


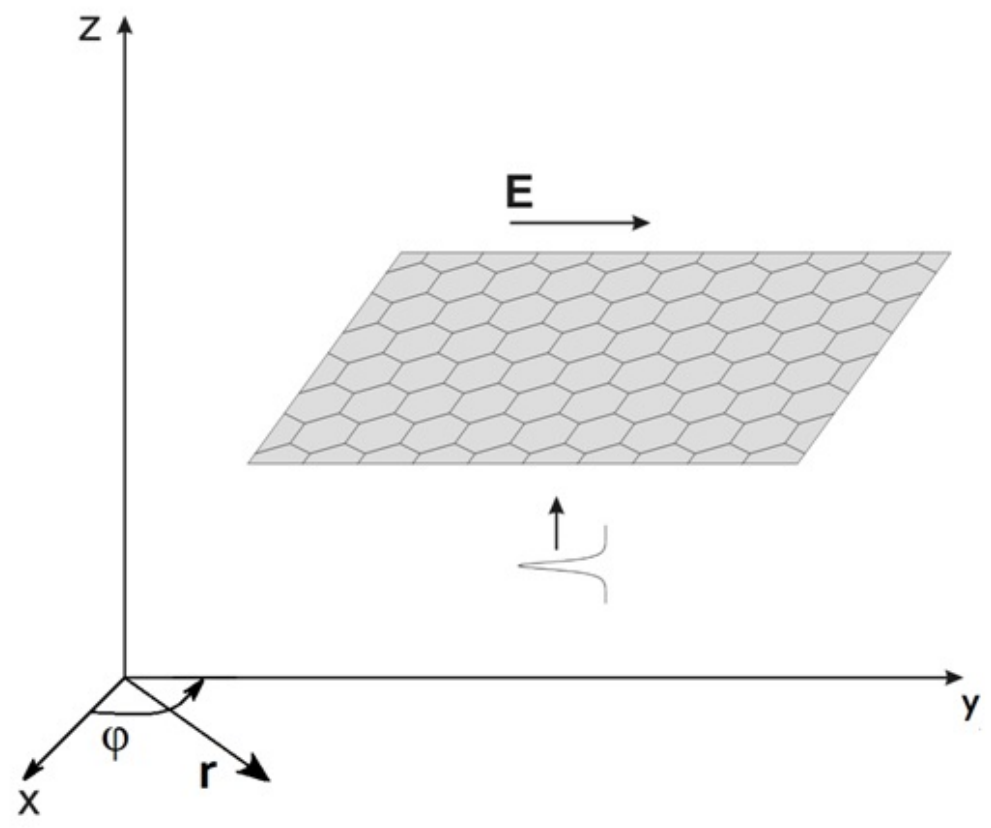

FIG. 1. Geometry of the problem

According to [9], we have:

$$
\nu_{y}=\nu\left(1+\frac{\alpha}{4} \ln \left(\frac{\Lambda+\sqrt{\Lambda^{2}+m^{2}}}{\sqrt{q_{x}^{2}+q_{y}^{2}}+\sqrt{q_{x}^{2}+q_{y}^{2}+m^{2}}}\right)\right),
$$

Here, $q_{x}, q_{y}$ - electron momentum component, $m$ - gap in the spectrum $(0.1 \mathrm{eV})$. It should be noted that the gap in the energy spectrum since graphene is considered with impurity.

As follows from the rules of quantum mechanics, in the presence of an external electric field of the $\mathbf{E}$ which for certainty is directed along the $\mathrm{x}$-axis, and will be hereafter considered in the calibration: $\mathbf{E}=-\partial \mathbf{A} / c \partial t$, pulse needs to be replaced on the generalized pulse: $\mathbf{q} \rightarrow \mathbf{q}-e \mathbf{A} / c$ ( is the electron charge).

We write the wave equation for the three-dimensional case in the cylindrical coordinate system:

$$
A_{t t}=\frac{1}{r} \frac{\partial}{\partial r}\left(r \frac{\partial A}{\partial r}\right)+\frac{\partial^{2} A}{\partial z^{2}}+\frac{1}{r^{2}} \frac{\partial^{2} A}{\partial \phi^{2}}+4 \pi j(A)
$$

Next, everywhere we believe that by virtue of the cylindrical symmetry: $\frac{\partial}{\partial \phi} \rightarrow 0$.

From the law of charge conservation, we have: $\rho \propto \tau \frac{j}{l_{y}}$ (here $\rho$ is the charge density, $\mathrm{j}$ is the current density along $y$ axis, $\tau$ is the electric field duration, $l_{y}$ is the characteristic length of the electric field pulse along the $y$ axis) shows that the duration of ESP has a significant impact on the accumulated charge, which creates an additional electric field. This electric field is interfering with the field of ESP. Previous estimates made by the authors (the stored charge is about $1-2 \%$ of the charge, giving contribution to the current) allow us to conclude that the charge accumulation effect for femtosecond pulses may be neglected. This is confirmed by numerical experiments for the case of carbon nanotubes and a pulse duration of tens of femtoseconds [8].

We write down the standard expression for the current density:

$$
\mathbf{j}=e \sum_{q} v_{y}\left(\mathbf{q}-\frac{e}{c} \mathbf{A}(x, y, z, t)\right)\left\langle a_{q}^{+} a_{q}\right\rangle
$$

where the brackets denote averaging with the non-equilibrium density matrix $\rho(t):\langle B\rangle=S p(B(0) \rho(t))$. Given that $\left[a_{q}^{+} a_{q}, H\right]=\left[n_{q}, H\right]=0$ (since the interaction with the external field is given by: $\varepsilon\left(\mathbf{q}-\frac{e}{c} \mathbf{A}(x, y, z, t)\right) a_{q}^{+} a_{q}$, $A=(0, A(x, y, z, t), 0), n_{q}$ is the number of particles) from the equations of motion for the density matrix immediately obtain that: $\left\langle a_{q}^{+} a_{q}\right\rangle=\left\langle a_{q}^{+} a_{q}\right\rangle_{0}$, where $\langle B\rangle_{0}=S p(B(0) \rho(0))$. Thus, in the expression for the current density, the number of particles can be used, which follows from the Fermi-Dirac distribution. Next, we 
consider the case of low temperatures, when only a small region in momentum space near the Fermi level gives a contribution to the sum (integral). Accordingly, we write the formula (3) as:

$$
\mathbf{j}=e \int_{-\Delta}^{\Delta} \int_{-\Delta}^{\Delta} d \mathbf{q}_{x} d q_{y} v_{y}\left(q-\frac{e}{c} \mathbf{A}(x, y, z, t)\right) .
$$

The region of integration over the pulses in (6) is determined from the condition of equality the number of particles:

$$
\int_{-\Delta}^{\Delta} \int_{-\Delta}^{\Delta} d q_{x} d q_{y}=\int_{Z B} d q_{x} d q_{y}\left\langle a_{q x, q y}^{+} a_{q x, q y}\right\rangle,
$$

the integration of the right is carried out over the first Brillouin zone.

The equation on the distribution of extremely short pulses can be written as:

$$
\begin{aligned}
& A_{t t}=\frac{1}{r} \frac{\partial}{\partial r}\left(r \frac{\partial A}{\partial r}\right)+\frac{\partial^{2} A}{\partial z^{2}}+\frac{1}{r^{2}} \frac{\partial^{2} A}{\partial \varphi^{2}}+4 \pi \Phi(A) \cdot f(z) \\
& f(z)=1-\alpha \cdot \exp \left(-\left(\frac{z-z_{0}}{\delta}\right)^{2}\right)
\end{aligned}
$$

here $\Phi(A)$ is determined by integrating in (5), $f(z)$ is the function that determines the spatial inhomogeneity, $\alpha$ is the depth of spatial inhomogeneity (when $\alpha=0$ - without inhomogenity), $\delta$ is the width of the spatial inhomogeneity, $z_{0}$ is the given position offset inhomogeneity.

\section{Results of numerical simulation}

Equation (6) was solved numerically [12]. The initial condition is chosen in the form:

$$
\begin{aligned}
& A(z, r, 0)=Q \exp \left(-\left(\frac{z-z_{0}}{\gamma_{z}}\right)^{2}\right) \exp \left(-\left(\frac{r}{\gamma_{r}}\right)^{2}\right) \\
& \frac{d A(z, r, 0)}{d t}=2 Q v_{z} \frac{z-z_{0}}{\gamma_{z}} \exp \left(-\left(\frac{z-z_{0}}{\gamma_{z}}\right)^{2}\right) \exp \left(-\left(\frac{r}{\gamma_{r}}\right)^{2}\right),
\end{aligned}
$$

where $r$ is the radius, $Q$ is the amplitude, $\gamma_{z}, \gamma_{r}$ are the determine the pulse width, $v_{z}$ is the initial pulse velocity along z-axis $z_{0}$ is the initial displacement of the pulse center. This initial condition corresponds to what is an extremely short pulse consisting of a single oscillation of the electric field applied to the sample. The values of energy parameters are expressed in units of $\Delta$. It should be noted that time evolution is variable.

The arising evolution of the electromagnetic field during its propagation through each sample is shown in Fig. 2.
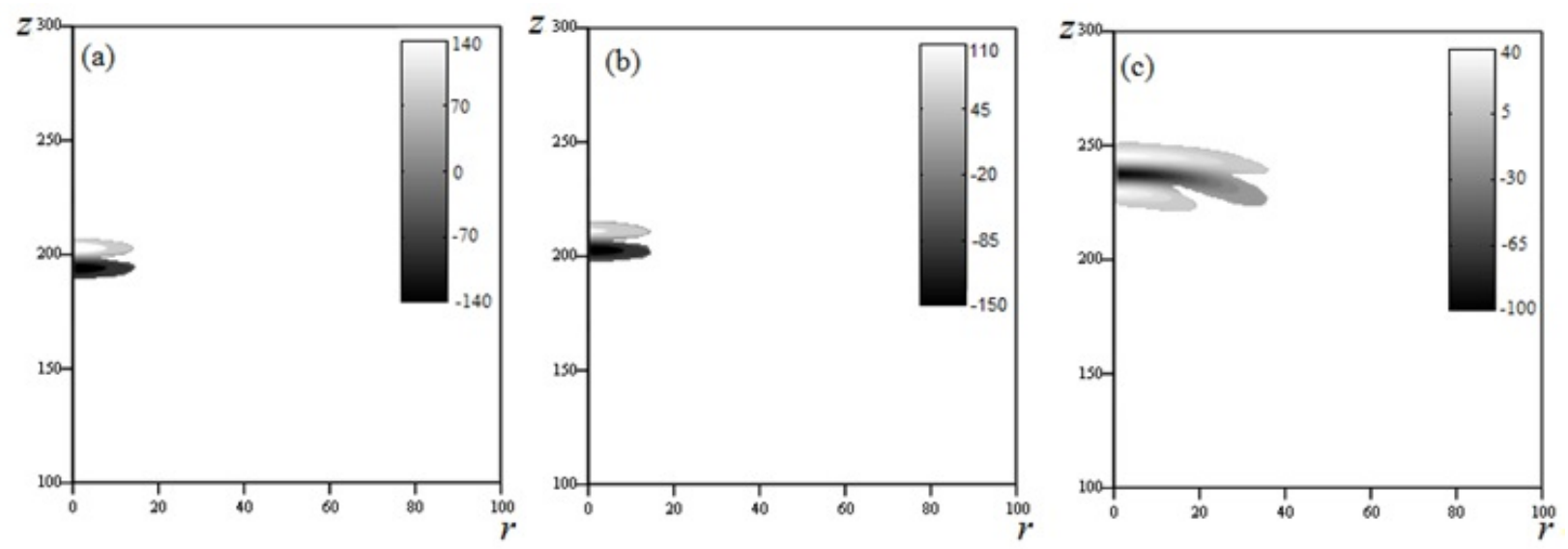

FIG. 2. The intensity of the three-dimensional electromagnetic pulse at different time points: (a) the initial pulse shape; (b) $t=1.0 \cdot 10^{-13} \mathrm{~s}$; (c) $t=5.0 \cdot 10^{-13} \mathrm{~s}$ (unity along $r$ and $z$ corresponds to $5 \mathrm{~nm}$ ) 
This pulse behavior is associated with the nonlinearity in (6). There is a decline in pulse amplitude, as well as its spreading over time. This allows us to draw conclusions regarding the instability of pulses, which leads to the disruption of their structure.

Dynamics of a three-dimensional extremely short pulse considering inhomogeneity of the environment is shown in Fig. 3.
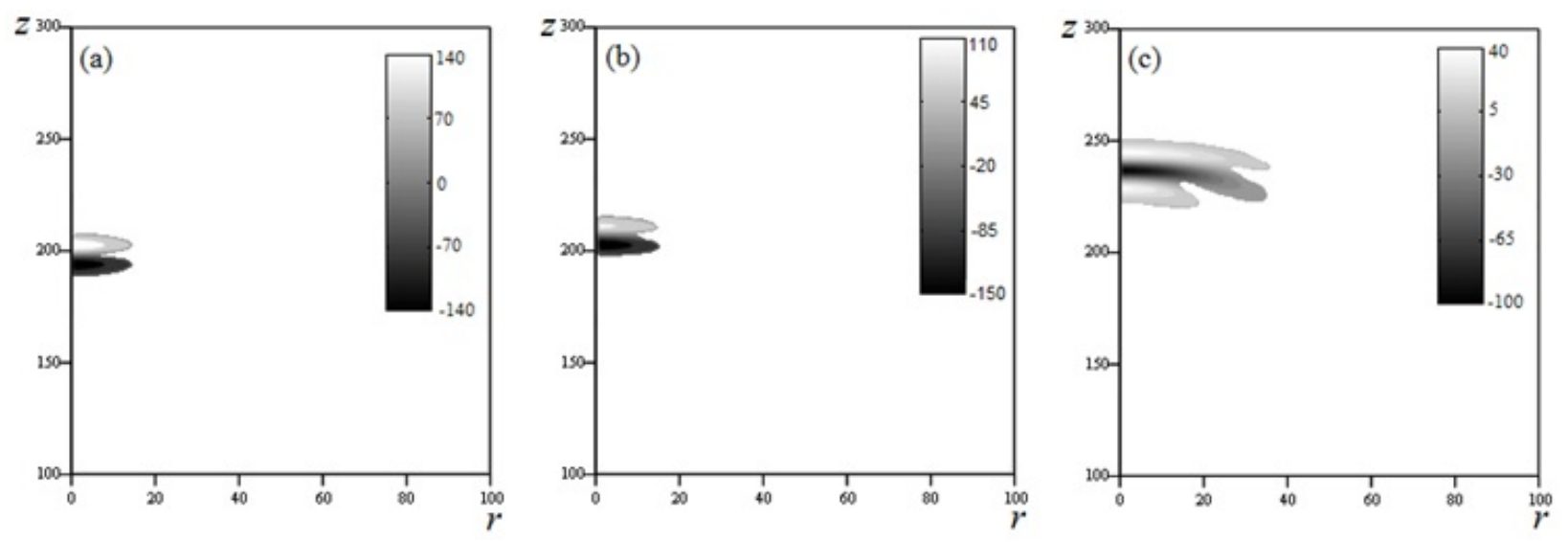

FIG. 3. The intensity of the three-dimensional electromagnetic pulse at different time points ( $\alpha=0.8$ r.u., $\delta=5$ r.u.): (a) the initial pulse shape; (b) $t=1.0 \cdot 10^{-13} \mathrm{~s}$; (c) $t=5.0 \cdot 10^{-13} \mathrm{~s}$ (unity along $r$ and $z$ corresponds to $5 \mathrm{~nm}$ )

As follows from Fig. 3, the introduction of spatial inhomogeneity does not preclude spreading of the pulse along the transverse coordinate.

Comparison of cases with consideration of the spatial inhomogeneity and without it is shown in Fig. 4.
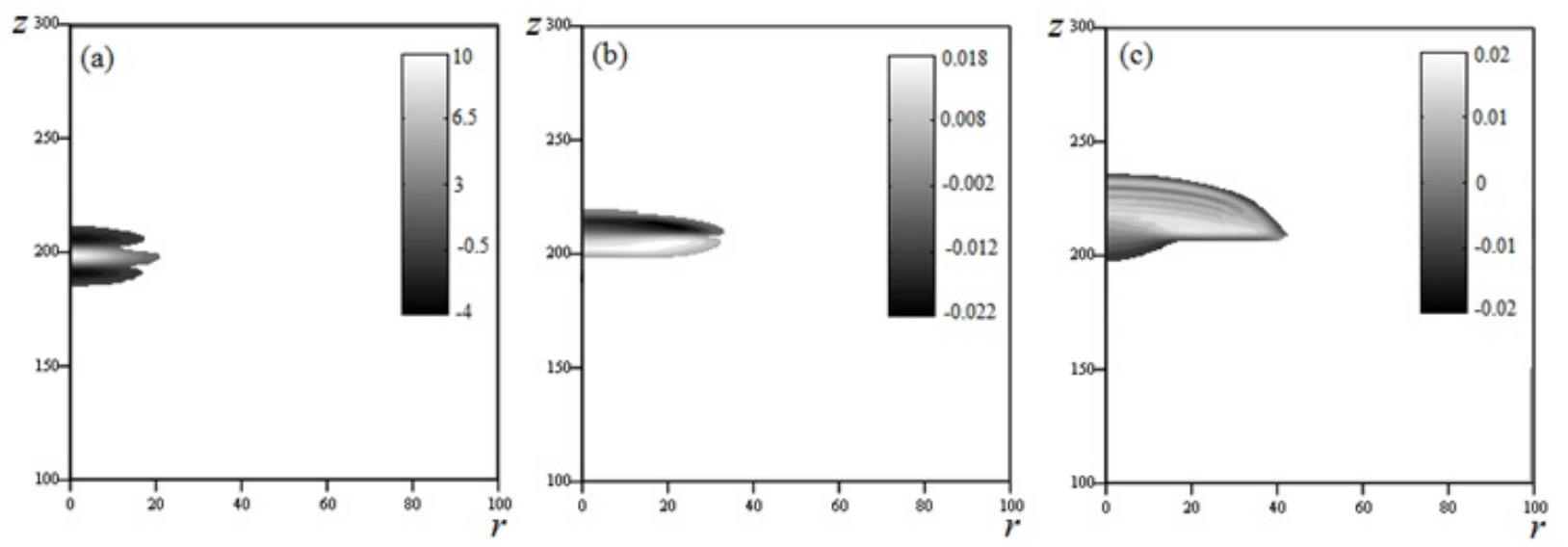

FIG. 4. The difference of the intensities of the three-dimensional electromagnetic pulse in the case of considering non-uniformity and without it: (a) the initial pulse shape; (b) $t=1.0 \cdot 10^{-13} \mathrm{~s}$; (c) $t=5.0 \cdot 10^{-13} \mathrm{~s}$ (unity along $r$ and $z$ corresponds to $5 \mathrm{~nm}$ )

As is shown by the results of numerical calculations, with the passage of time, the difference between the pulse shape in the homogeneous recording case and without it becomes less noticeable. But, in the case for the introduction of spatial non-uniformity in the environment, the momentum in the amplitude is reduced by about $0.05 \%$ in comparison with the case of a homogeneous medium.

We also note that we studied the impact of inhomogeneity of parameters $\alpha$ and $\delta$ on the form of extremely short pulses. Numerical calculations have shown that the depth and width of the input inhomogeneity has little effect on the shape of the pulse.

Practical application of the methodology proposed in the article is the following: all the obtained nanotubes and graphene contain impurities that affect the propagation process of extremely short optical pulses due to electron density inhomogeneity. Thus, by examining the evolution of such pulse features, we can identify the characteristics of the impurities. 


\section{The conclusions}

In conclusion, we will now formulate the main outcomes of this work:

(1) Three-dimensional extremely short optical pulses in doped graphene propagate unstably with violation of the pulse shape.

(2) Introduction of spatial inhomogeneities in the medium does not permit significant reduction of the pulse losses in amplitude in comparison with the case of a homogeneous medium.

(3) The depth and width of the spatial inhomogeneity does not seem to have much influence on the shape of the pulse.

\section{Acknowledgements}

This work was supported by the Russian Foundation for Basic Research under project No. 16-32-00230, by the Ministry of Education and Science of the Russian Federation project no. MK-4562.2016.2.

\section{References}

[1] Akhmanov S.A., Vysloukhy V.A., Chirikin A.S. Optics of femtosecond laser pulses. New York, AIP, 1992.

[2] Belonenko M.B., Demushkina E.V., Lebedev N.G. Electromagnetic soliton in bundles of carbon nanotubes. Physics of the Solid State, 2008, 50, P. 383-389.

[3] Yanyushkina N.N., Belonenko M.B., Lebedev N.G., Zhukov A.V., Paliy M. Extremely Short Optical Pulses in Carbon Nanotubes in Dispersive Nonmagnetic Dielectric Media. Int. J. Mod. Phys. B, 2011, 25, P. 3401-3408.

[4] Belonenko M.B., Popov A.S., Lebedev N.G., Pak A.V., Zhukov A.V. Extremely short optical pulse in a system of nanotubes with adsorbed hydrogen. Phys. Lett. A, 2011, 375, P. 946-952.

[5] Belonenko M.B., Fedorov E.G. Extremely short electromagnetic pulses in an array of carbon nanotubes with a longitudinal field inhomogeneity. Physics of the Solid State, 2013, 55, P. 1333-1337.

[6] Belonenko M.B., Nevzorova Ju.V., Galkina E.N. Few cycle pulses in the Bragg medium containing carbon nanotubes. Nanosystems: physics, chemistry, mathematics, 2014, 5(5), P. 644-649.

[7] Konobeeva N.N., Belonenko M.B. Propagation of femtosecond pulses in carbon nanotubes. Nanosystems: physics, chemistry, mathematics, 2014, 5(1), P. 91-97.

[8] Zhukov A.V., Bouffanais R., Fedorov E.G., Belonenko M.B. Three-dimensional electromagnetic breathers in carbon nanotubes with the field inhomogeneity along their axes. J. Appl. Phys., 2013, 114, P. 143106.

[9] Suzuura H., Ando T. Crossover from symplectic to orthogonal class in two-dimensional honeycomb lattice. Phys. Rev. Lett., 2002, 89, P. 266603.

[10] Belonenko M.B., Konobeeva N.N. Ultrashort Electromagnetic Pulses in Graphene with Disorder. Optics and Spectroscopy, 2015, 119(2), P. 82-85.

[11] Kotov V.N., Pereira V.M., Uchoa B. Polarization charge distribution in gapped graphene: Perturbation theory and exact diagonalization analysis. Phys. Rev. B, 2008, 78, P. 075433

[12] Bahvalov N.S. Numerical methods (analysis, algebra, ordinary differential equations. Moscow, Nauka, 1975,632 p. 